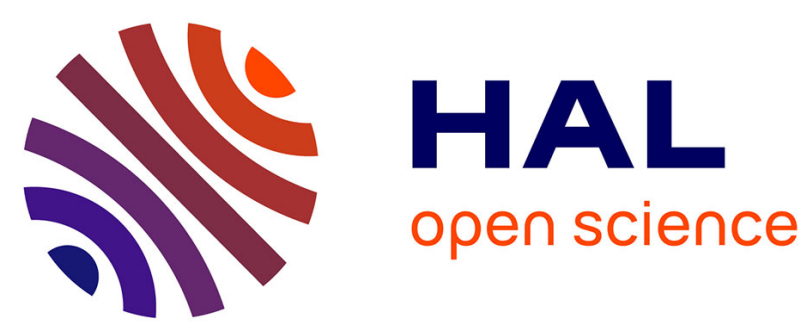

\title{
Integration of SCADA Services and Power-Hardware-in-the-Loop Technique in Cross-Infrastructure Holistic Tests of Cyber-Physical Energy Systems
}

van Hoa H Nguyen, Tung Lam Nguyen, Quoc Tuan T Tran, Yvon Besanger, Raphael Caire, Hoa Van, Lam Nguyen, Tuan L Nguyen

\section{To cite this version:}

van Hoa H Nguyen, Tung Lam Nguyen, Quoc Tuan T Tran, Yvon Besanger, Raphael Caire, et al.. Integration of SCADA Services and Power-Hardware-in-the-Loop Technique in Cross-Infrastructure Holistic Tests of Cyber-Physical Energy Systems. IEEE Transactions on Industry Applications, 2020, 56 (6), pp.7099-7108. 10.1109/TIA.2020.3021365 . hal-03260231

\section{HAL Id: hal-03260231 https://hal.science/hal-03260231}

Submitted on 20 Jan 2022

HAL is a multi-disciplinary open access archive for the deposit and dissemination of scientific research documents, whether they are published or not. The documents may come from teaching and research institutions in France or abroad, or from public or private research centers.
L'archive ouverte pluridisciplinaire HAL, est destinée au dépôt et à la diffusion de documents scientifiques de niveau recherche, publiés ou non, émanant des établissements d'enseignement et de recherche français ou étrangers, des laboratoires publics ou privés. 


\title{
Integration of SCADA services and Power-hardware-in-the-loop technique in cross-infrastructure holistic tests of cyber-physical energy systems
}

\author{
Van Hoa Nguyen, Tung Lam Nguyen, Quoc Tuan Tran, Senior member, IEEE, Yvon Besanger, Senior \\ member, IEEE, and Raphael Caire, Senior member, IEEE
}

\begin{abstract}
Cyber-Physical Energy System (CPES) - complex juxtaposition of multiple energy domains and communication and automation technologies - requires a sophisticated testing framework to achieve holistic assessment and validation, especially at large scale. In this paper, the real Supervision, Control and Data Acquisition (SCADA) system of CPES is integrated with the advanced techniques: Real-Time Simulation (RTS) and Powerhardware-in-the-loop (PHIL), in a cross-infrastructure manner to create a realistic validation environment for CPES. On the one hand, the method can be applied to extend the capacity of the infrastructures as well as creating a common resource and expertise pool. On the other hand, this approach combines the realistic data and advanced SCADA services with the flexibility of the real-time simulation platform, which provides the possibility to emulate extreme and faulty scenarios with virtual equipment and topology. The proposed approach is demonstrated via a case study of analysing the impact of communication on advanced voltage and frequency restoration in an isolated microgrid. The case study is implemented on two remote platforms PREDISPRISMES (70 km apart). The validation framework comprises the RTS and PHIL platform coupled with the OPC UA SCADA system in PRISMES, the control algorithm and the communication network simulator located in PREDIS platform.
\end{abstract}

Index Terms-Interoperability, SCADA-as-a-service, Crossinfrastructure, Hardware-in-the-loop, Co-simulation, Real-time simulation, Holistic Testing.

\section{INTRODUCTION}

I $\mathrm{N}$ order to achieve the decarbonisation scenario, the power system is modernized and is embedded with various Information and Communication Technologies (ICT) and automation solutions, including advanced metering infrastructure, big data assessment and cyber-security [1]. This juxtaposition of multiple technologies has transformed the power system into a cyber-physical energy system (CPES), featuring a strong interrelation and correlation of the individual domains [2]. A comprehensive and holistic validation strategy for CPES needs to harmonize distinct behaviours (continuous-discrete event)

This work is partly supported by the European Community's Horizon 2020 Program - under the projects "ERIGrid II" (Grant Agreement No. 870620) and "United Grid" (Grant Agreement No. 773717).

V.H. Nguyen and Q.T. Tran are with Univ. Grenoble Alpes, CEA, LITEN, INES, Department of Solar Technologies, F-73375 Le Bourget du Lac, France

Y. Besanger and R. Caire are with Univ. Grenoble Alpes, Grenoble INP, G2Elab, CNRS, F-38000 Grenoble, France.

T.L. Nguyen is with The University of Danang - University of Science and Technology, Danang, Vietnam. between the Informatics and Communication Technologies (ICT) and the energy systems (electrical grid, heating system) and various different time scales, from several $\mu s$ (electrical transient behaviour) to several days (market management). It requires a suitable complexity of validation environment, which is not always feasible in the framework of a single Research Infrastructure (RI). For large-scale CPES assessment, researchers are coupling multiple interoperable platforms [3][7] into a holistic experiment to allow the mutualisation of equipment (hence, reduction of investment) and the combination of expertise in different domains of CPES.

The classical validation workflow in CPES assessment is based on mainly two approaches: simulation and real hardware testing. Simulation provides the advantages of rapidity, flexibility and versatility while does not risk damaging the equipment. Real hardware testing requires much more time and investments and is hard to reconfigure in case of an adaptation, but it allows the consideration of real behaviour and impact of equipment. These individual methods, up to now, were used in a fragmented manner, allows the consideration of some certain aspects of the big picture with a limited interaction to the others, without any guarantee how the whole system would react to a solution (e.g. a local optimization may not lead to global optimization; how a single device failure may affect the network or how the communication performance influences the system stability, etc.). More advanced approaches to cope with the multi-domain natures of CPES are: co-simulation [1], real-time (RT) simulation [8], hardware-in-the-loop (HIL) and power-hardware-in-the-loop (PHIL) [9] or eventually the combination of both techniques [10]. The coupling of advanced techniques involving hardware (e.g. HIL/PHIL) over a long distance is, however, challenging due to the impact of latency to the accuracy and stability of the experiment, which may eventually damage the equipment under test [11].

An important aspect in the CPES system that is not sufficiently considered in current validation frameworks is the Supervision, Control and Data Acquisition (SCADA) system and its impacts on system performance. In a large scale CPES, the SCADA system can regroup and orchestrate all the ICT and automation solutions. In current CPES validation methods, the SCADA system, in particular, and the telecommunication aspects, in general, are mostly considered by integrating communication simulators [12], [13]. While this solution is 
cost-effective and allows the consideration of the interrelation of cyber-physical aspects, the SCADA system of CPES is a complex multi-layer system with industrial protocols and information models, which are not always supported by the communication simulators (mostly ICT oriented). Moreover, the realistic telecommunication characteristics such as sampling time of sensors, response time of controller, server capability, transition of different information models and protocol, redundancy of architecture (history and back-up servers) can't be fully included with current communication network simulators. They are, however, important for a correct and realistic assessment of advanced functionalities of CPES (distributed control, cyber-security, big-data analysis, etc.). On the other hand, the real SCADA system is often associated with a fixed physical system and does not provide enough flexibility for testing of CPES in different configurations and environments.

In this paper, we propose an approach for interoperability of SCADA services and RT simulation/HIL/PHIL of (remote) RI in a holistic platform for CPES validation and assessment. The integration of SCADA functionalities to the cross infrastructure holistic tests [14] via the SCADA-as-a-service architecture [15] introduces realistic data and SCADA functionalities in real-time to the CPES validation framework while the integration of RTS/HIL enables the consideration of CPES taking into account multiple domains and time scales, as well as real equipment behaviour. This approach allows the configuration of complex and sophisticated validation framework for CPES using realistic data and functions from the SCADA system in flexible or extreme scenarios managed by RTS/HIL.

The approach is demonstrated via a case-study of voltage and frequency restoration in isolated microgrid, implemented in a coupled infrastructure of PREDIS (Grenoble INP) and PRISMES (CEA Ines) platforms, located $70 \mathrm{~km}$ apart; including RT simulation, OPC UA SCADA system and PHIL in a holistic test setup.

The rest of the paper is organized as follows. Section II presents the approach of cross-infrastructure holistic testing for smart grid and the interoperability requirements for such test. The integration between the SCADA system and the RTS/HIL platform (among multiple RI) is considered in section III. In section IV, the proposed method is demonstrated via the crossinfrastructure holistic test-case. Section V concludes the paper and outlines possible future directions.

\section{CROSS-INFRASTRUCTURE HOLISTIC TESTING FOR SMART GRID AND INTEROPERABILIY REQUIREMENTS}

CPES are multi-domain systems and due to their diverse and complex natures, the implementation of their validation framework is challenging:

- The integrity and the interdependency of the subsystems in different domains need to be well reflected in the validation framework.

- A local solution obtained by a single domain test may not be (or compatible with) the global solution.

- Expertise from multiple domains is required to fully characterize and to implement a holistic framework.

Combining complementary expertise and platforms of RI in a holistic test is, therefore, a judicious solution that does not require heavy investments in time, infrastructure and human resources. To successfully integrate different actors together in a holistic framework (e.g. SCADA system, RT simulation platform and different kinds of equipment), it is necessary and imperative to achieve interoperability among them. When the holistic test involves combining assets from several platforms, an inter-platform interoperability is also required for their connection and integration. Achieving interoperability also facilitates the connection and integration of new platforms to the group and reduces the cost of installation of experimental modules and integration processes.

\section{A. Interoperability requirements}

In [16], we proposed a five-layer interoperability architecture for a holistic CPES test (Fig. 1). A holistic experiment integrates information and data from various sources with different formats (via cosimulation or via platform coupling). Depending on the level of coupling that one wishes to achieve in the framework, it is necessary to achieve interoperability up to a certain abstraction layer. The practical setup and harmonization between different parties involved in the test need to be taken care of accordingly.

- Interoperability at technical implementation level involves a harmonization of communication infrastructure (i.e. physical layer - e.g. intra/internet, common data sheet, etc.). This low level allows the exchange of signals (e.g. binary, hexadecimal, etc.) between the parties

- The dynamic data level involves the harmonization of communication protocols (e.g. TCP/IP, UDP, Lora, etc.) among parties. It also decides the synchronization aspects (e.g. emission and reception time, multicast, etc.), encoding/decoding methods (e.g. Big Endian, Little Endian) and data format (e.g. 8 bits, etc.). Interoperability at this layer ensures the good transmission of data (e.g. v value) among the parties.

- Syntactic formalism level relates to the methods of interpretation of data (i.e. information model ). Interoperability at this level allows translating correctly the received data into meaningful information (e.g., the received $\mathrm{v}$ value is the voltage at bus A).

- Semantic level describes the interrelations among the information of an entity or among entities in a scenario (e.g. $\mathrm{i}$ and $\mathrm{v}$ are current and voltage of bus $\mathrm{A}$; bus $\mathrm{A}$ is connected with bus $\mathrm{B}$ and $\mathrm{C}$, etc.). Interoperability at syntactic and semantic levels allows the correct and automatable processing of the scenario.

- Finally, the conceptual scenario level describes the multi-domain system configuration (e.g. interconnection power/ICT/heating, etc.), and the desired test scenario (e.g. generator $\mathrm{x}$ starts at time $\mathrm{t} 1$, load $\mathrm{y}$ trips at time $\mathrm{t} 2$, etc.). Interoperability at this level allows the participating parties to execute their parts and to contribute to the global test scenario correctly.

In general, the integration of SCADA system to the holistic framework involves mostly the three lower layers. While the practical implementation of interoperability at physical and dynamic levels are quite straightforward (i.e. implementing 


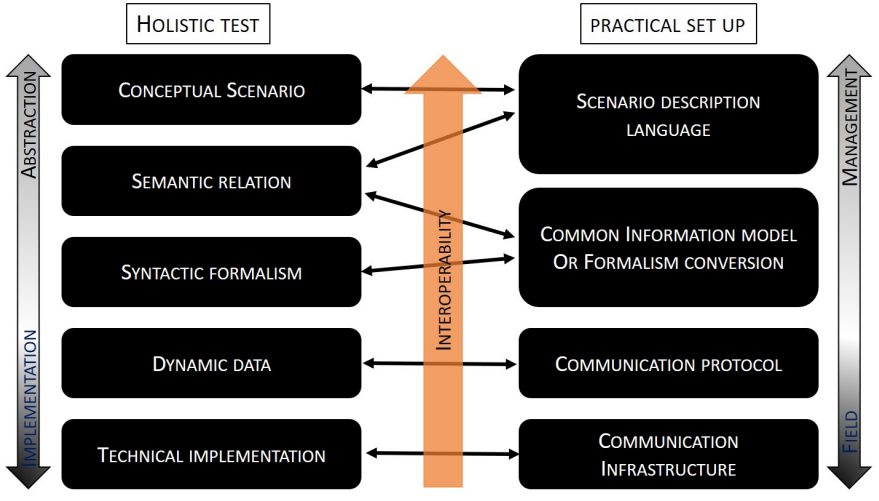

Fig. 1: Interoperability architecture and practical setup requirements.

common communication protocols or employing a protocol conversion interface). It is not the case for the three higher abstraction levels. In general, the syntactic and semantic convention of the power system are achieved via employing a common information model (e.g. CIM (IEC 61970/61968) [17], Multispeak [18], IEC 61850 dynamic data model [19] or OPC UA address space [20], etc.). The existing information models are however domain oriented and may not fully cover the inter-domain links (e.g. between ICT-Power or between the electrical grid and the heating system). This gap can be fixed via the inclusion of a scenario description language (standard TTCN-3 [16], TPLAN [21] and the 62559 template from SGAM model [22]) which also enables the implementation of interoperability at the conceptual level. For instance, there are automated or semi-automated methods for implementing popular information models (e.g. CIM [23]), converting and communicating between different information models (e.g. CIM and OPC UA [24]). The combination of power system's information models and scenario description languages is unfortunately not easy to achieve, let alone to automate the process.

\section{B. Practical Implementation of Interoperability}

In case one desires to include the power system SCADA system, as proposed in [15], it is imperative for the participating parties to exchange and to comprehend correctly the system description and the scenario (i.e. to reach at least syntactic interoperability). This task is however challenging because the traditional SCADA system does not support the concept of the information model. Data is processed and stored syntactically only with advanced systems (i.e. object-oriented data model). In case that the SCADA system does not support information model implementation, additional human effort is necessary for harmonization (e.g. pre-agreed convention of naming scheme, message format and communication protocol, etc.). On the other hand, the integration of more advanced SCADA system to a holistic framework in the syntactic layer requires potentially a harmonization and adaptation of the SCADA's information model to the one employed in the framework, or vice versa.
In fact, the physical implementation of SCADA system of infrastructure depends strongly on:

- the field-level technical choices of the system operator: communication infrastructure (e.g. Ethernet, power line communication-PLC, Wi-Fi, etc.), protocols (e.g. IEC61850, DLMs, etc.), information models (e.g. CIM, Multispeak, etc.) and environments (e.g. Labview, etc.),

- the topological interrelation of its elements (centralized, distributed, hybrid)

- and the actual need for application functionalities (availability, time steps, stability, etc.).

The suggestions for implementations at different levels of interoperability are presented in a generic manner because the specific technical choices would depend on a real situation (e.g. the SCADA's information model, the employed protocols, etc.) and maybe not trivial in another situation.

Implementing interoperability including SCADA system can be done via several levels. At the most basic level (i.e. technical interoperability), the communication infrastructure among parties needs to be integrated. We focus on the following implementation at the dynamic and syntactic level which are required for the integration of the SCADA.

1) Interoperability of Dynamic Data: At this level, the participating parties exchanges dynamic data (e.g. measurement, set points, etc.). These data may not be related to another by default. The interrelations among them (i.e. syntactic and semantic levels), which are necessary to execute the holistic test accurately, are achieved via pre-agreed convention (e.g. common naming scheme, system architecture diagram) and may require human intervention.

An important issue in implementing interoperability at the dynamic level is the transition among various communication protocols. CPES hosts a wide range of protocols from field devices (e.g. Modbus, DNP3, ZigBee, etc.) to the internet (e.g. https), via Local Area Network (LAN) or long-distance communication (e.g. LoRa, GSM, 4G, etc.). Each protocol specifies a different message format. The complexity is often degraded by the variety of operating systems (OS - e.g. UNIX, LINUX, etc.), which demand different memory order (e.g. 3264 bits, big-Endian or little-Endian, etc.).

In general, OPC and OPC UA can be used for the transition from platform-dependent and component-based field level protocol to SCADA system and other interoperable applications. Besides acting as a translator, OPC UA can also bridge the gap between the local network and wide area services [25].

2) Interoperability of Information Models: In general, it is ideal to implement a common information model for all participating parties in the holistic scenario. However, the SCADA system of each partner is issued from its system configuration and architecture, as well as the development environment and it would be costly to switch. Even if a platform does not possess a SCADA system supporting information model, its data is often organized with its own naming and format convention, which may differ from one partner to another. Most of the time, interoperability in a holistic scenario is achieved with harmonization of those "semi" information models. 

level.

The adaptation can be done at the syntactic level or semantic

- If the SCADA system is integrated as "black box" and no topology information is required, then a simple naming scheme and message format harmonization suffices for syntactic level.

- However, if the topology of the considered system matters during the integration, then semantic interoperability is required and that information needs to be extracted and imported. For instance, CIM allows the representation of topological information in the form of CIM/XML (eXtensible MarkUp Language)/RDF (Resource Description Framework). This description can be used to configure the semantic communication in the framework or to set up the intermediate server for information exchange [23].

These implementations can be done over the base of interoperability at the communication level.

\section{INTEROPERABILITY OF SCADA SERVICES WITH REAL-TIME SIMULATION AND (P)HIL PLATFORM}

Interoperability of SCADA services with RTS and (P)HIL in a holistic platform (Fig. 2) bring out several benefits:

- Possibility to consider the scenario with real data from the expected system environment (including uncertainties that are not readily available with a simple simulation model: e.g. PV/wind installation angle, diversity of meteorological condition, number of users in a building, etc.) and advanced SCADA services.

- The RTS can complement the physical platform with virtual equipment that is not available.

- RT interface between the SCADA system and the RTS allows the replication and the consideration of real equipment in a virtual scenario, which is sometimes extreme or faulty (e.g. for protection or anti-islanding testing), without damaging the real devices.

- The (P)HIL capability allows local consideration of real equipment in their deployment setup, interacting with the virtualized CPES (e.g. harmonic injection, anti-islanding capability, etc.).

\section{A. Communication Implementation}

The coupling setup between the SCADA system and the RT platform depends on the architecture of the system and the test-scenario. Generally, the RT platform can be coupled to:

- field devices level (via component-dependent protocols), RTU/PLC level (via IEC 61850/Modbus protocol),

- OPC level (via OPC or OPC UA protocol)

- or via application level (via UDP/TCP).

The actual setup should be chosen as a function of the required services from the SCADA system and with the criterion of minimizing the latency, e.g. if the RT platform requires measurements from a single device (e.g. RTU), it can be coupled to that RTU via Modbus, instead of going through the upper SCADA layers. The choice is also limited by the communication capability of the RT simulator (i.e.

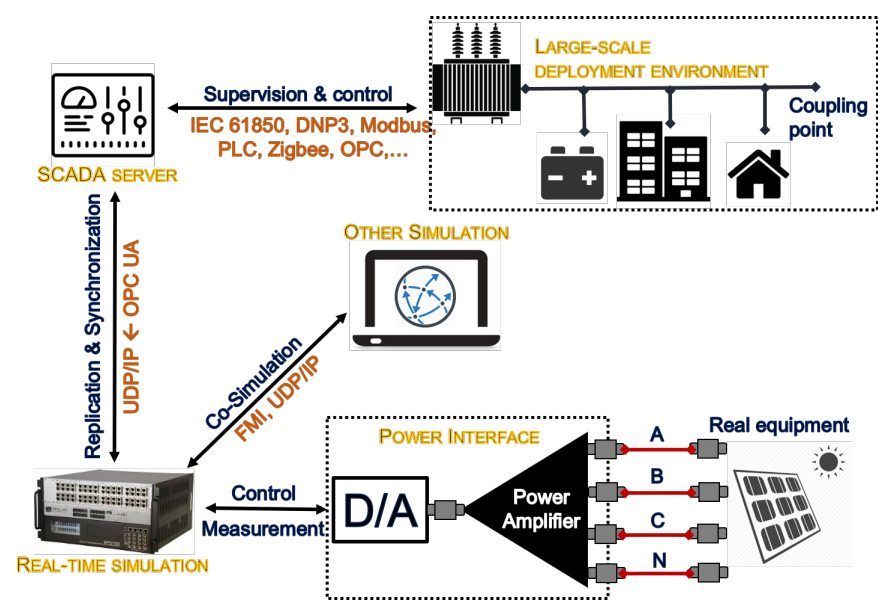

Fig. 2: Integration of SCADA system with RTS and PHIL platform.

supported protocols). Several implementation methods can be considered:

- Via a software interface that converts, (dynamic or steady) data from the SCADA side's protocol to the RTS's protocol (e.g. Modbus to https). This approach is easy to implement (dynamic level interoperability), but lacks support for system semantics and requires more efforts when the system becomes large scale.

- Via a common database (e.g. SQL, Redis, etc.) that serves as an exchange point. This common database can also be configured to arrange the received data to a defined information model (syntactic and semantic level interoperability).

- In the particular case when the required SCADA service is provided by an external actor (e.g. PV production forecasting function developed in Matlab providing prediction to the SCADA system). Then the coupling point (RT platform - SCADA) can be set as the point where the SCADA acquires the service (e.g. a .xml file).

\section{B. Synchronization - Latency Compensation}

RT simulation, HIL and PHIL require the simulator to satisfy rigorous constraints on timing and determinism, because the "virtual" elements need to keep up with the real-time behaviour, which is particularly challenging due to the capacity of the simulator and due to the potential variability of time steps. Moreover, latency in the Power-hardware-in-the-loop setup may cause the lead to instability and may cause damage to the equipment [10].

The coupling of the SCADA system and the RTS/HIL platform introduces into the framework the latency between them as well as the limitations of physical equipment (sampling time, response time, etc.). While they do not prevent the functionality of the interface (i.e. information exchange between the actors), the results may consist disturbances (e.g. sample from a later step may arrive before its precedent samples) and may not reflect a realistic scenario (e.g. the emulators running shortened profiles coupled with real devices). 
For synchronization, we can choose to set up the RT platform and the SCADA system to exchange with the time steps satisfying the conditions proposed in [25]. This condition ensures that the exchanged value could be assigned to the same sync step of the other actors (i.e. the order of signal is well preserved), while capturing all the variation and providing a "closest to reality" information to the RT/HIL platform. The last criterion to choose the sync step is the requirement of the considered application (e.g. control generally requires much faster sampling time than monitoring).

Finally, several compensation methods can be considered to compensate for the latency between the SCADA system and the RT/HIL platform or between the RI:

- The exchanged signals are "shifted" through timefrequency transformation [26]. The advantage of this approach is that it works well with signals with harmonics.

- An observer is constructed to estimate the state of the subsystem subjected to data exchange, based on the measurements of previous steps and the space state of the subsystem [27].

- A similar method using linear prediction for latency compensation is registered in [28]. This method performs linear prediction directly on the exchanged signal and does not require knowledge on the subsystem space state.

All of the existing methods share the two shortcomings: firstly, the latency is assumed to be known. The exact estimation of this latency is however problematic, especially in geographically distributed interoperability involving clock synchronization. Secondly, none of the existing methods can handle well signals with micro-variation, which is very common with real measurements. For RMS signal, they all perform reasonably; however, the performance degrades significantly when we go into sub-second scenarios.

In any case, the latency should be compensated only if it is subsequently introduced by the test-setup. When the latency is a part of the desired scenario (e.g. latency between RTU and field devices), then it should be kept as it is for a realistic consideration.

\section{CASe-STUdy: CROSS-INFRAstructure IMPLEMENTATION OF VOLTAGE AND FREQUENCY RESTORATION IN ISOLATED MICROGRID}

In this section, the proposed approach is demonstrated via a case study of advanced voltage and frequency restoration in an isolated microgrid. The case study is implemented on two remote platforms PREDIS-PRISMES (70 km apart). The validation framework comprises the RTS and PHIL platform coupled with the OPC UA SCADA system in PRISMES, the control algorithm and the communication network simulator located in PREDIS platform.

The considered case study involves the restoration of voltage and frequency in a PV supported micro-grid. In this case study, we are interested mainly in several aspects:

- Validation (good functionality) of the control.

- Demonstration of a holistic test-case, where virtual/real equipment can impact each other in an experimental setup closely representing the desired scenario.

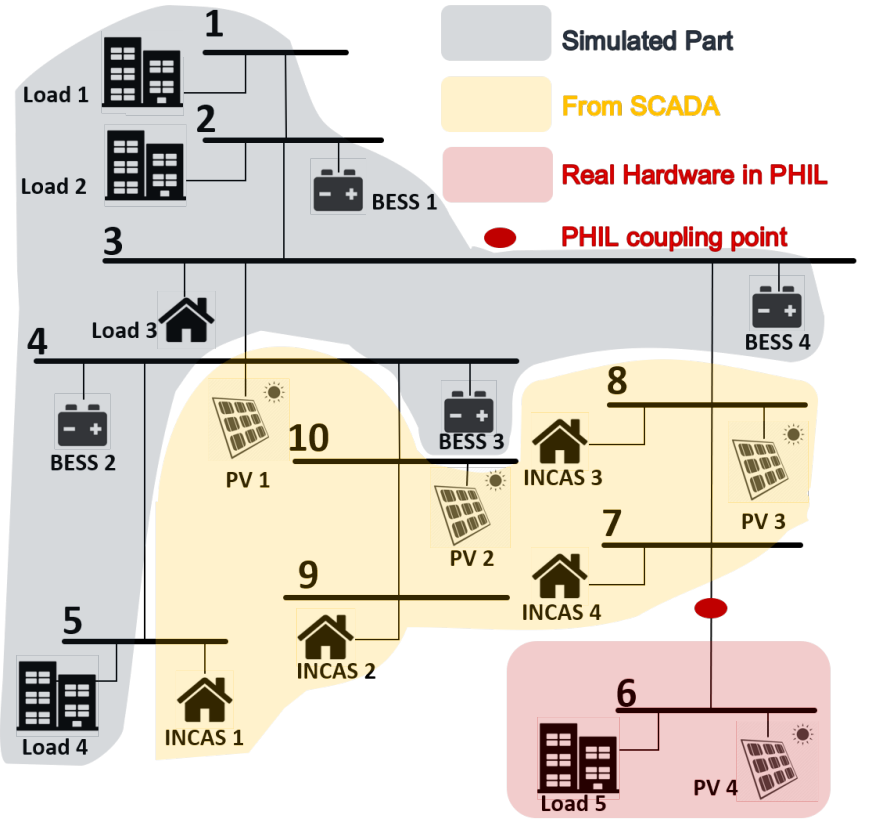

Fig. 3: The microgrid configuration considered in the test-case.

- Demonstration of our approach to combine resources and technical expertise from different research infrastructures.

- Demonstration of the interoperability of SCADA system and RT simulation.

\section{A. Test-Case Description and Cross-Infrastructure Set Up}

1) Test-Case Description: We consider in this test-case the microgrid (MG) supported by four PV packs and four battery energy storage systems (BESS), supplying nine loads; as depicted in Fig. 3. The MG operates in the islanded mode without the support from the main grid. The stability of the grid is guaranteed by the cluster of battery energy storage systems (BESSs). Each BESS has a local controller which operates in parallel with the MG central controller (MGCC) (Fig. 4). A typical hierarchical control structure is applied to control the MG under the fluctuation of loads and PVs power. The aim is to maintain the system frequency and voltage at the references value and to ensure the proportional power-sharing among BESSs.

At primary level, the local droop control of each BESS reacts rapidly to balance power between consumers and suppliers against disturbances:

$$
\begin{gathered}
f-f^{r e f}=-k_{P}\left(P-P^{r e f}\right) \\
V-V^{r e f}=-k_{Q}\left(Q-Q^{r e f}\right)
\end{gathered}
$$

where $k_{P}$ and $k_{Q}$ are the droop coefficient; $f-f^{r e f}$ and $V-V^{\text {ref }}$ are the grid frequency and the voltage deviation respectively; $P-P^{r e f}$ and $Q-Q^{r e f}$ are the variations in the active and reactive power delivered by the BESSs to compensate such deviations.

The primary control may cause the deviation of frequency and voltage even in the steady-state. The secondary control is then activated to bring the frequency and voltage to the 


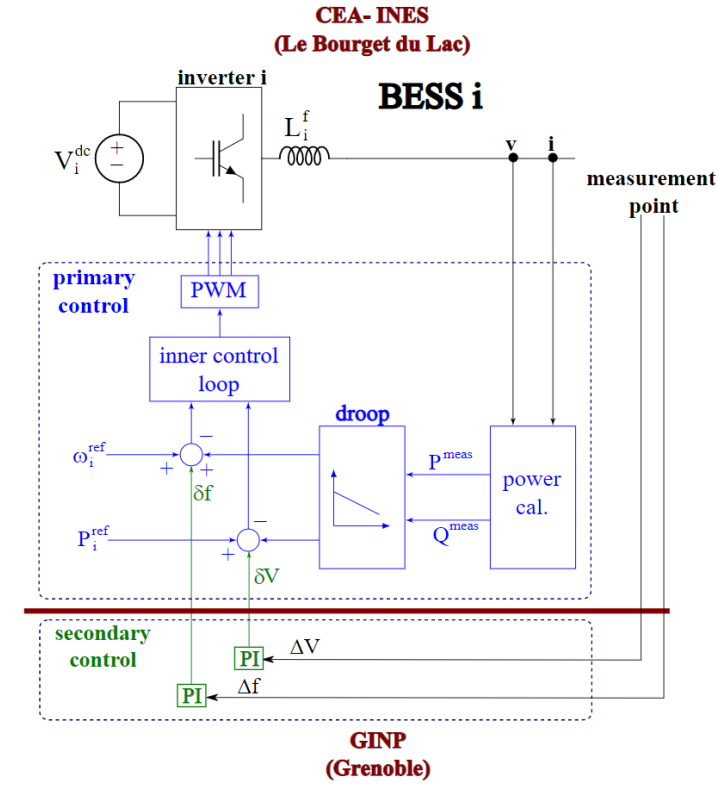

Fig. 4: Control diagram of a BESS.

reference values. The coordination between local controllers is required to avoid the conflict of control signals. In this study, we use a centralized approach for secondary control purposes. The MGCC collects measurements and distributes the control signals as follows:

$$
\begin{gathered}
\delta f=k_{I f} \int\left(f-f^{r e f}\right) \\
\delta V=k_{I V} \int\left(V-V^{r e f}\right)
\end{gathered}
$$

where $k_{I f}$ and $k_{I V}$ are the control parameters.

Fig. 4 illustrates the control diagram of the $i^{\text {th }}$ BESS with two control layers: the local control for primary control purpose and the central controller which takes in charge of secondary control. The secondary control receives voltage and frequency signals from a common measurement point. In this work, the secondary control is in a MGCC located in a long distance site and will broadcast control signals to all BESS local controllers.

The time delays when transferring signals between control layers may lead to voltage and current fluctuation [29]. A MG with the hierarchical control structure might have weak stability or even instability due to the influence of time delay. The stability analysis considering network delay has been presented theoretically in previous works [30] based on small signal stability, Lyapunov theory and linear matrix inequalities technique. The control gains in the central secondary control need to be tuned to adapt network communication condition. In this work, we investigate the time delay dependency in a more practical way with the proposed platform.

2) Cross-infrastructure Integration of SCADA - PHIL: The test case is implemented in two parts (Fig. 5), combining the resources and expertise of two partners: CEA INES (power system - electrical manipulation) and Grenoble INP (control - communication network simulation).

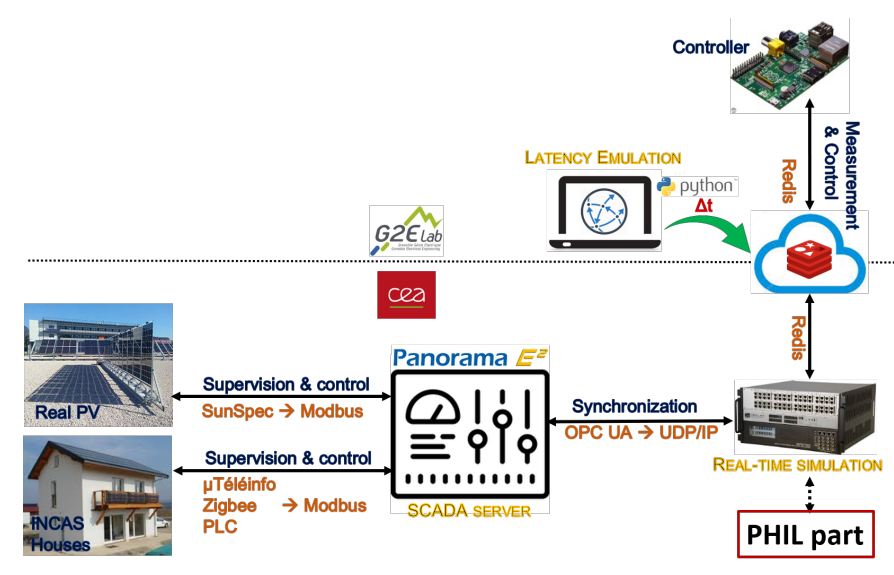

Fig. 5: The multi-site platform setup.

(i) The power part of the MG is located in the PRIMES platform (CEA INES, Chambéry, France). One PV is the hardware device interfaced with the real-time simulator in the PHIL set up, while the three remaining PVs are emulated in OPAL-RT with real-time synchronized data from the SCADA system. One load is a hardware device (Cinegia load) while other loads are synchronized with the onsite building consumption. The droop controllers are located locally for fast reactive time.

(ii) The MGCC is developed and located at PREDIS platform (Grenoble INP, Grenoble, France - $70 \mathrm{~km}$ apart from CEA-INES) based on the secondary control law in (3) and (4).

In this case study, we consider the real PV production and load consummation in the MG. For that purpose, we create a link with the SCADA system of CEA INES to get these measurements in real-time and to inject them to the $\mathrm{MG}$ simulated in the RT simulator OPAL RT. The smart houses INCAS and the PV packs (each with 20-60 Si-Monocrystalline panels and PV inverters) are physical hardware (Fig. 6) and are synchronized to the test via the OPC UA SCADA Panorama E2 of PRISMES platform in CEA. In term of model, those PV and load are emulated as current sources with synchronized active and reactive power.

To examine the behaviour of the control algorithm (voltagefrequency deviation) to real devices, the PV pack and load at bus 6 is then replace with the real load Cinegia 30kVA and the real PV inverter SMA $17 \mathrm{kVA}$ connected with a PSI PV emulator. These real equipments are physically connected to the MG via a PHIL interface (Fig. 7). The PHIL Interface follows Ideal Transformation Method (ITM).

Since bus 6 consists of inverter-based source and load and has a single point of coupling (PCC) with respect to the considered grid, it is necessary to validate the anti-islanding capacity of the PV inverter in case of grid disconnection. IEC 62116 [31] requires the PV inverter to check the loss of the grid and stop supplying the power (i.e. to disconnect within $2 \mathrm{~s}$ ) to the closely matched demand by the loads (with the tolerance of $\pm 5 \%-10 \%$ ). The trip time is calculated based on the IAC values in an islanded condition. This scenario is particularly hard to detect for traditional passive based 


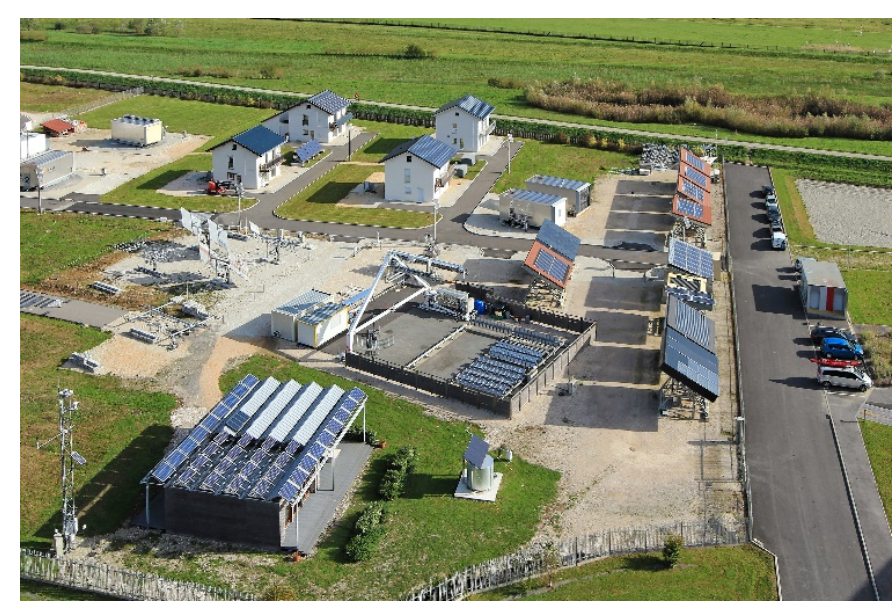

Fig. 6: The INCAS houses and the PV packs at PRISMES that were considered in the test-case.

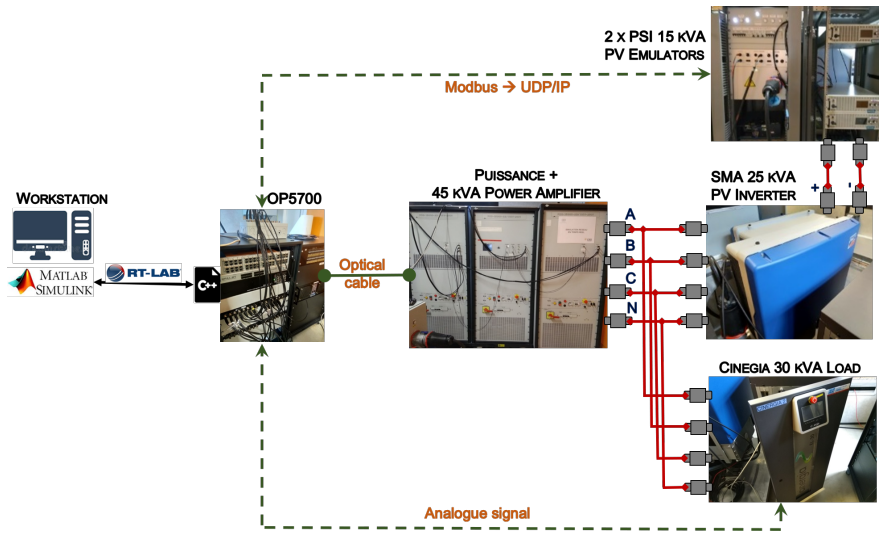

Fig. 7: The PHIL part setup.

protection, due to the difficulty of setting admissible parameter range. For the validation of anti-islanding capability, the PSI PV emulators are then calibrated to be well within $\pm 5 \%$ of the demand by the Cinegia (Fig. 8). The cluster is then "balanced" and there is almost no power exchange with the other parts ( Iexchange $_{\text {en }} \sim 0$.

The communication between two partners is configurated through a Redis server working as a private cloud database. Communication emulators at Grenoble INP observes the real communication between two site and are capable of emulating artificial communication scenarios between the MGCC and the MG (e.g. latency, noise, congestion, etc.) to investigate the influence of communication network on the control performance.

\section{B. Experimental Results}

The parameters and droop coefficients of BESS local controllers are shown in Table I.

Three cases will be considered corresponding to three different communication network scenarios. We validate firstly the functionality of the control in the actual setup, and then we investigate the capacity of the control in a more extreme communication scenario (high latency). Finally, communication with noise is then considered.

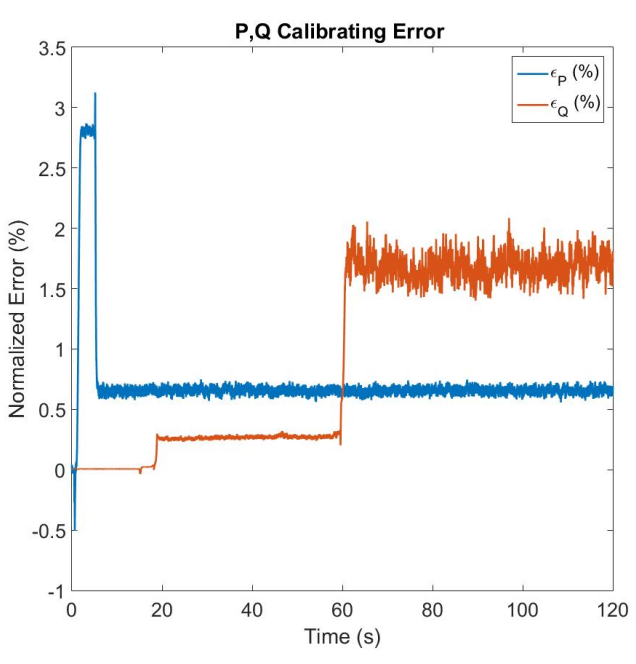

Fig. 8: PQ Calibrating error for the islanding situation at bus 6.

TABLE I: Droop control parameters.

\begin{tabular}{|c|c|c|c|}
\hline \multicolumn{2}{|c|}{ BESS-1 and BESS-2 } & \multicolumn{2}{c|}{ BESS-3 and BESS-4 } \\
\hline \hline$k_{1}^{P}, k_{2}^{P}$ & $4 \mathrm{e}-5$ & $k_{3}^{P}, k_{4}^{P}$ & $2 \mathrm{e}-5$ \\
\hline$k_{1}^{Q}, k_{2}^{Q}$ & $2 \mathrm{e}-3$ & $k_{3}^{Q}, k_{4}^{Q}$ & $1 \mathrm{e}-3$ \\
\hline$L_{1}^{f}, L_{2}^{f}$ & $0.4 m H$ & $L_{3}^{f}, L_{4}^{f}$ & $0.4 m H$ \\
\hline
\end{tabular}

1) Case 1: Normal condition: The latency between the two sides of the platforms is measured to be consistently around $32 \mathrm{~ms}$, with some occasional peaks of $85 \mathrm{~ms}$. The integral control gains are chosen as a trade-off between tolerable communications delay and convergence speed:

$$
k_{I f}=2, k_{I V}=5
$$

The measurement of the active power of BESSs, frequency and voltage at the inverter outputs are shown in Fig. 9.

Initially, the local droop controllers regulate the power outputs of BESSs to ensure the stability and keep the system at the steady-state. However, there exist the errors of frequency and voltages in comparing with the normal state. After that, we trigger the secondary controller remotely, the controller gradually compensates the deviation and recovers frequency and voltage to desired values. The controller works correctly to deal with the disturbances of loads and the fluctuation of PV systems.

2) Case 2: Bad Communication Scenario: In this case, we add $500 \mathrm{~ms}$ to the latency between the MGCC and the local entities to investigate the behaviour of the controller in a bad communication scenario (i.e. longer distance or slower protocols). With the same control parameters in Case 1, when the secondary start, the controller failed to stabilize the MG as illustrated in Fig. 10. The selection of the integrator gain value is no longer correct for this environment network. It demonstrates the huge impact of the communication network to the system operation if it is not taken into account right in the design phase.

At 98.38 s the voltage surpasses the limit of $15 \%$ raise and 

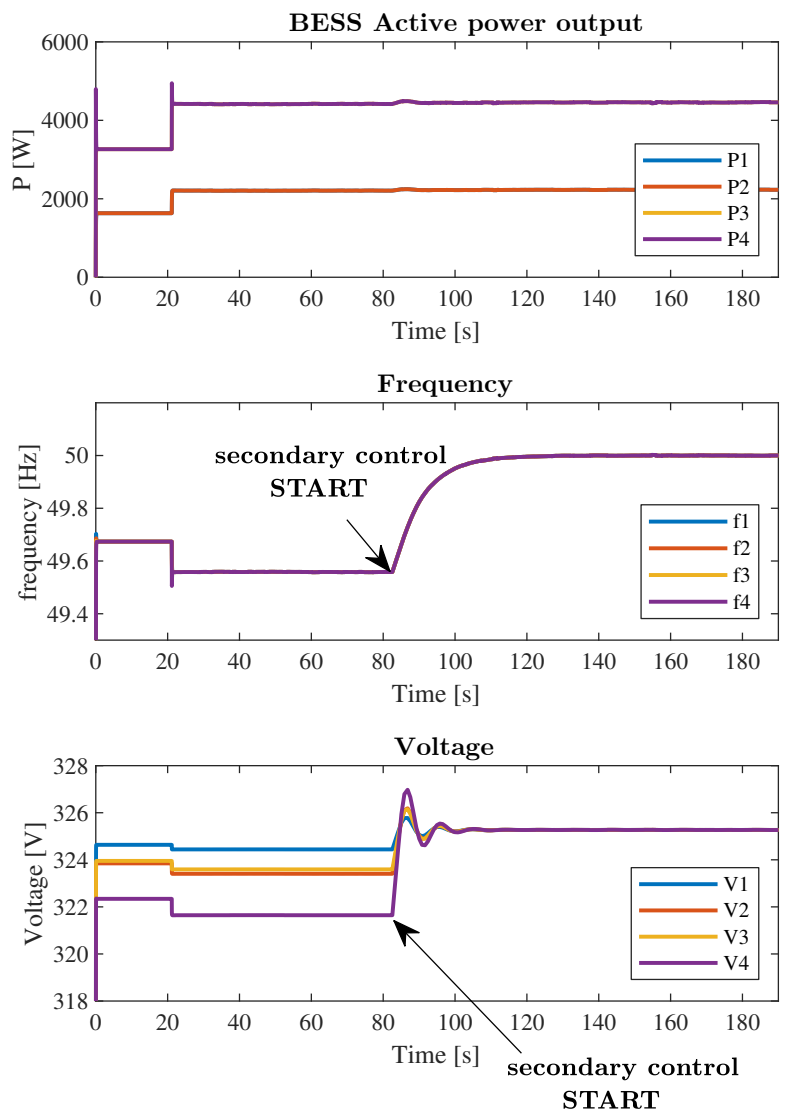

Fig. 9: Case 1: without additional latency, $k_{I f}=2, k_{I V}=5$.

does not return to the allowed tolerance in the required time ( $2 \mathrm{~s}$ - this time span may vary from one grid code to another). The overvoltage triggered the protection at the physical point coupling and disconnected bus 6 . Due to this sudden voltage drop, the PV current supplying the load flows through the PCC for a brief moment before the PV inverter stops. The time taken by the inverter to check the loss of the grid and stop supplying the power to the closely matched demand by the loads is 0.108 s (i.e. time before IAC returns to 0 - Fig. 11 ), which is well within the limit of $2 \mathrm{~s}$ required by IEC 62116.

The controller, therefore, needs to be modified to adapt to the new working condition. The gains are reduced as follows:

$$
k_{I f}=0.5, k_{I V}=0.5
$$

Fig. 12 displays the results with the updated controller. When the secondary control is activated, the frequency and voltages are restored to the references. The power quality is maintained even with a considerable step change of loads or different operational states of BESSs (charge/discharge).

3) Case 3: Communication With Noise: We consider, in this case, the performance of the control algorithm under communication noise. The parameter is then reconfigured as in Case 1. An additive Gaussian noise (variance $\sigma^{2}=0.01$ ) is added to the communication between the controller and the microgrid. The power sharing among the BESS, the voltage and frequency are then shown in Figure 13. It demonstrates
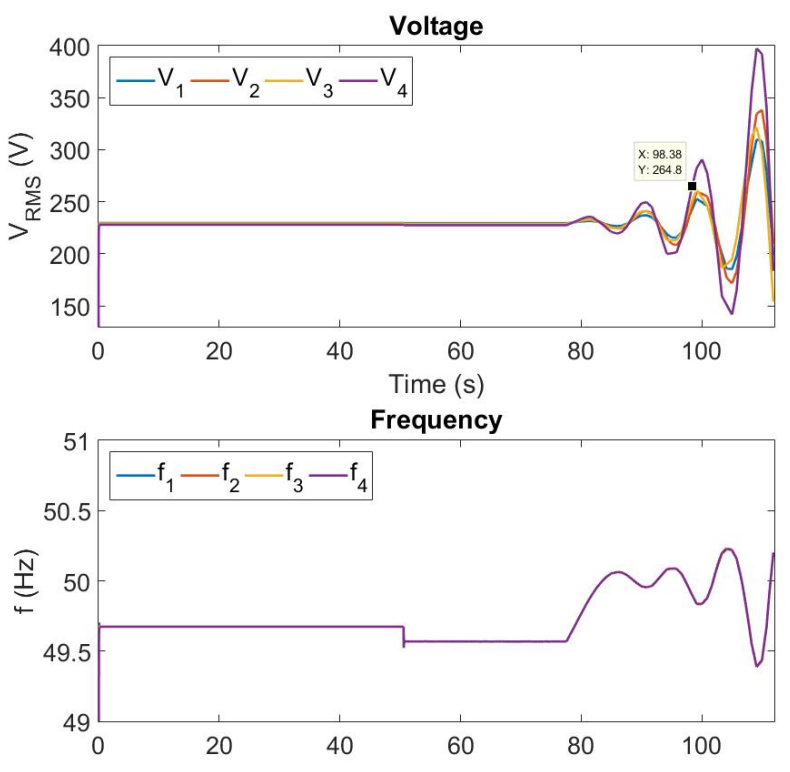

Fig. 10: Case 2: with additional latency, $k_{I f}=2, k_{I V}=5$.

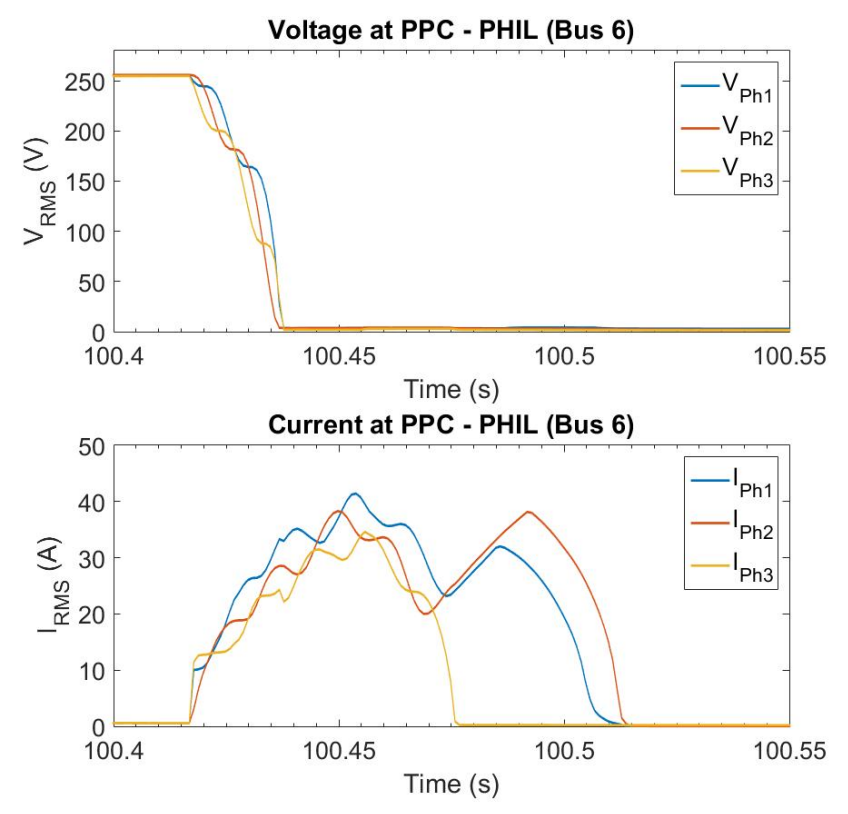

Fig. 11: Anti-Islanding activation at bus 6 .

that the control can withstand communication noise to a certain extent.

The considered test case demonstrates a holistic approach to consider a CPES in its complete form with all the interaction and interdependency between cyber and physical parts. This consideration led to the early reconfiguration of the control according to the deployment environment, right in the design phase. Indeed, in this demonstrating test case, we are capable to observe clearly how the communication performance influences the electrical microgrid behavior, triggers anti-islanding protection on physical inverter and how we could use the 

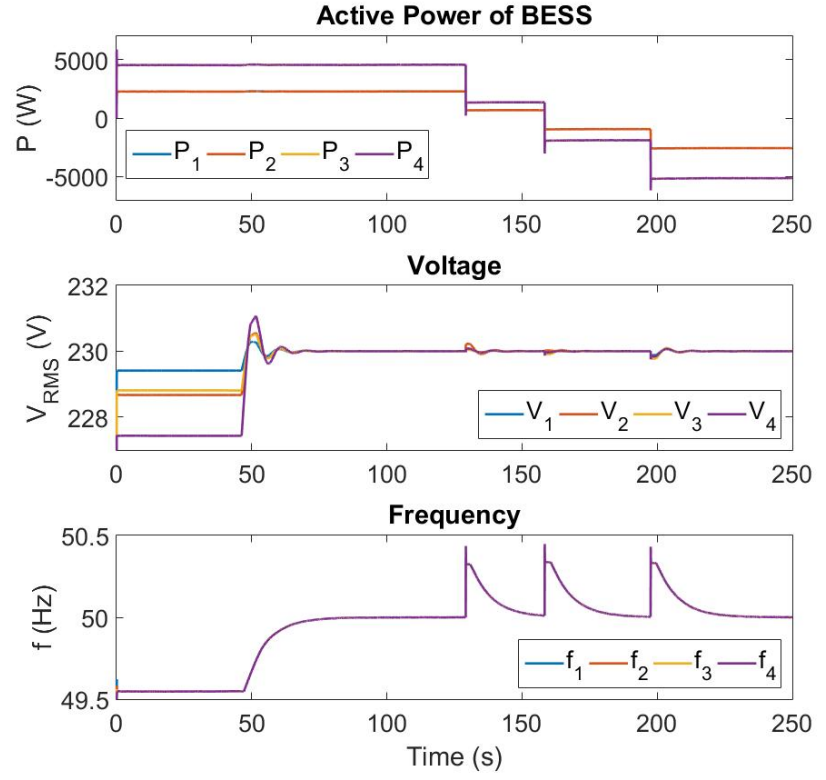

Fig. 12: Case 2: with additional latency, $k_{I f}=0.5, k_{I V}=0.5$.
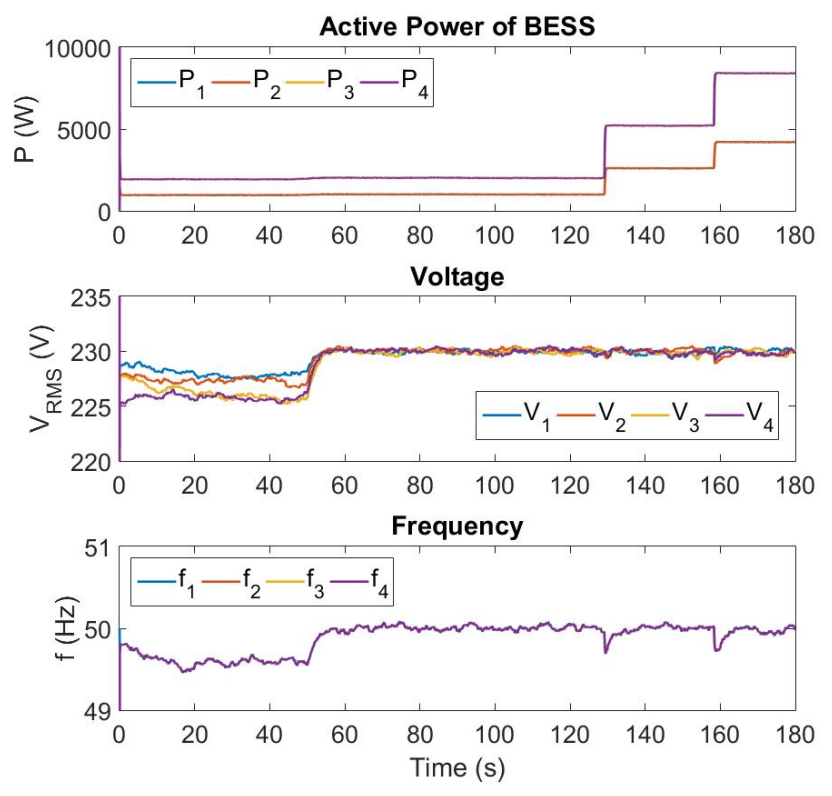

Fig. 13: Case 3: Control under communication noise.

results to tune the control parameters.

\section{CONCLUSION}

In this paper, we proposed a method to combine SCADA services, simulation and Power-hardware-in-the-loop, in a cross-infrastructure manner, to create a holistic validation framework for cyber-physical energy system solutions. Using a combination of these fragments to create a holistic and realistic environment, we can achieve transient validation on physical components via localized PHIL experiment; coupled with the desired CPES generated in real-time simulation with SCADA service coupling. Moreover, the method is designed in a vision of cross-infrastructure interoperability, which allows the combination of infrastructures (and the expertise to manipulate the infrastructure) from multiple partners in to a large-scale but holistic CPES validation framework. It helps to save time and money (for investment and formation) and more importantly, to close the gap between the design and the industrial deployment of a solution.

In this vision, the advancements of this approach can be summarized as following:

- The validation framework is closer to reality thanks to the synchronization with SCADA service to create digital twins of physical elements (e.g. PV arrays, building, etc.).

- The multi-domain nature of CPES and the interaction among them are addressed via co-simulation.

- Local and transient behavior of the integrated DRES can be considered in the most realistic condition with real devices (via PHIL interface) and the propagation of its impact at a global level can be considered in the holistic environment.

- Finally, with cross-infrastructure interoperability, expertise and infrastructure from different partners can be combined to examine a large scale holistic CPES.

The proposed method improves the realism of the testing environment, enables the possibility of building large scale CPES testing setup, complements the drawbacks of the existing technologies, without requiring heavy investments in time, infrastructure and human resources.

\section{REFERENCES}

[1] C. Steinbrink et al., "Simulation-based validation of smart grids-status quo and future research trends," in International Conference on Industrial Applications of Holonic and Multi-Agent Systems, Lyon, France, Aug. 2017.

[2] A. A. van der Meer et al., "Cyber-Physical Energy Systems Modeling, Test Specification, and Co-Simulation Based Testing," Pittsburgh, PA, USA, May 2017.

[3] F. Lehfuss et al., "Coupling of Real-Time and Co-Simulation for the Evaluation of the Large Scale Integration of Electric Vehicles into Intelligent Power Systems," Belfort - France, Dec. 2017.

[4] M. Stevic et al., "Multi-site European framework for real-time cosimulation of power systems," IET Generation, Transmission \& Distribution, vol. 11(17), 2017.

[5] B. Lundstrom et al., "Evaluation of system-integrated smart grid devices using software- and hardware-in-the-loop," in 2016 IEEE Power Energy Society Innovative Smart Grid Technologies Conference (ISGT), 2016, pp. $1-5$.

[6] F. Marten et al., "Result processing approaches for large smart grid cosimulations," in Computer Science - Research and Development, 2017, 2017.

[7] Y. Wang et al., "A Distributed Control Scheme of Microgrids in Energy Internet and Its Multi-Site Implementation," IEEE Transactions on Industrial Informatics, pp. 1-1, 2020, conference Name: IEEE Transactions on Industrial Informatics.

[8] IEEE, "PES Task Force on Real-Time Simulation of Power and Energy Systems, Real-time Simulation Technologies for Power Systems Design, Testing, and Analysis," IEEE Power and Energy Technology Systems Journal, vol. 2, no. 2, Jun. 2015.

[9] G. Lauss et al., "Characteristics and Design of Power Hardware-in-theLoop Simulations for Electrical Power Systems," IEEE Transactions on Industrial Electronics, vol. 63, no. 1, pp. 406-417, Jan. 2016.

[10] V. H. Nguyen et al., "Using Power-Hardware-in-the-loop Experiments together with Co-simulation in a holistic approach for cyber physical energy system validation," in Proceeding of the IEEE PES International Conference on Innovative Smart Grid Technologies IEEE ISGT Europe 2017, 2017. 
[11] V. H. Nguyen et al., "Real-Time Simulation and Hardware-in-the-Loop Approaches for Integrating Renewable Energy Sources into Smart Grids: Challenges \& Actions," in InProceeding of the IEEE PES International Conference on Innovative Smart Grid Technologies IEEE ISGT Asia 2017, 2017.

[12] P. Palensky et al., "Co-Simulation of Intelligent Power Systems Fundamentals, software architecture, numerics, and coupling," IEEE Industrial Electronics Magazine, vol. 11, no. 1, Mar. 2017.

[13] IEEE PES Task Force on interfacing techniques for Simulation Tools, "Interfacing Power System and ICT Simulators: Challenges, State-ofthe-Art and Case Studies," IEEE Transaction on Smart Grid, vol. PP, no. 99, 2016.

[14] V. H. Nguyen et al., "Integration of SCADA services in crossinfrastructure holistic tests of cyber-physical energy systems," in Inproceedings of the IEEE EEEIC 2019 Conference, Genoa, Italia, Jun. 2019.

[15] V. H. Nguyen, Q. T. Tran, and Y. Besanger, "SCADA as a service approach for interoperability of micro-grid platforms," Sustainable Energy, Grids and Networks, vol. 8, pp. 26-36, Dec. 2016.

[16] V. H. Nguyen, Y. Besanger, Q. T. Tran, and T. L. Nguyen, "On Conceptual Structuration and Coupling Methods of Co-Simulation Frameworks in Cyber-Physical Energy System Validation," Energies, vol. 10, no. 12, p. 1977, Nov. 2017.

[17] International Electrotechnical Commission, "Application integration at electric utilities - System interfaces for distribution management Part 14: Multispeak - CIM Harmonization,” TC 57 - Power system management and associated information exchange, Tech. Rep., 2013.

[18] A. Gary, R. Greg, and R. Gerald, "MultiSpeak and IEC 61968 CIM: Moving Toward Interoperability," in Grid-Interop Forum, Atlanta, GA, 2008.

[19] International Electrotechnical Commission, "IEC61850 - Power Utility Automation," TC 57 - Power system management and associated information exchange, Tech. Rep., 2003.

[20] Electrical Power Research Institute, "OPC Unified Architecture - Part 1: Overview and concepts," TC 65/SC 65E - TR 62541-1:2010, Tech. Rep., 2010.

[21] E. T. S. Institute, "Methods for Testing and Specification (MTS); TPLan: A notation for expressing Test Purposes," ESTI Standard ETSI ES 202 553 V1.2.1, Jun. 2009.

[22] CEN-CENELEC-ETSI, Smart Grid Coordination Group, Smart Grid Reference Architecture (SGAM), v 3.0. November, 2012.

[23] M. T. Le et al., "CIM compliant multiplatform approach for cyberphysical energy system assessment," Auckland, New Zealand, Dec. 2017.

[24] S. Rohjans, K. Piech, M. Uslar, and J. Cabadi, "Cimbat - automated generation of cim-based opc ua-address spaces," in 2011 IEEE International Conference on Smart Grid Communications (SmartGridComm), 2011, pp. 416-421.

[25] V. H. Nguyen, T. L. Nguyen, Q. T. Tran, and Y. Besanger, "Synchronization conditions and Real-time constraints in co-simulation and Hardware-in-the-Loop techniques for Cyber-Physical Energy System assessment," Sustainable Energy, Grids and Networks, 2019.

[26] E. Guillo-Sansano, A. Roscoe, and G. Burt, "Harmonic-by-harmonic time delay compensation method for Phil simulation of low impedance power systems," Sep. 2015, pp. 560-565.

[27] J. L. Cale et al., "Mitigating Communication Delays in Remotely Connected HArdware-in-the-Loop Experiments," IEEE transactions on industrial electronics, vol. 65, no. 12, pp. 9739-9747, 2018.

[28] R. Liu et al., "Geographically distributed real-time digital simulations using linear prediction," International Journal of Electrical Power \& Energy Systems, vol. 84, pp. 308-317, Jan. 2017.

[29] C. Dong et al., "Time-Delay Stability Analysis for Hybrid Energy Storage System With Hierarchical Control in DC Microgrids," IEEE Transactions on Smart Grid, vol. 9, no. 6, pp. 6633-6645, Nov. 2018.

[30] L. Jiang et al., "Delay-Dependent Stability for Load Frequency Control With Constant and Time-Varying Delays," IEEE Transactions on Power Systems, vol. 27, no. 2, pp. 932-941, May 2012.

[31] I. E. Commission, "Utility-interconnected photovoltaic inverters - Test procedure of islanding prevention measures IEC 62116:2014," Tech. Rep. IEC 62116:2014, 2014.

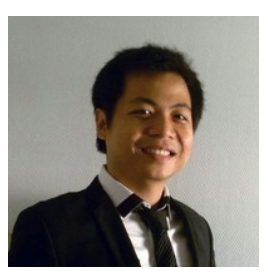

Van-Hoa Nguyen received the M.S. degree and Engineering diploma in Mechatronics from Grenoble INP, France in 2010 and the Ph.D. degree from INSA Lyon, France in 2014. He is currently a researcher at the Alternative Energies and Atomic Energy Commission (CEA) - National Institute for Solar Energy (INES). His research interests include interoperability, holistic validation of cyber-physical energy system and hardware-in-the-loop.

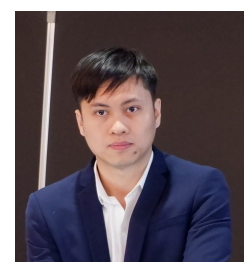

Tung-Lam Nguyen received the Master's degree in electrical engineering from National Taiwan University of Science and Technology, Taiwan, in 2014, and the Ph.D. degree from the University of Grenoble Alpes, Grenoble, France, in 2019. He is currently a Lecturer with the Faculty of Electrical Engineering, University of Science and Technology, The University of Danang, Vietnam. His research interests include distributed control and optimization, interoperability and co-simulation in smart grid.

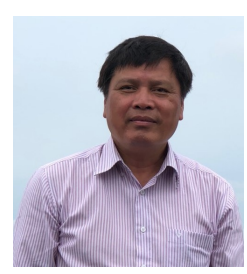

Quoc-Tuan Tran received his Ph.D. degree in Electrical Engineering from the Grenoble Institute of Technology in 1993. He is currently a Professor with INSTN - Paris Saclay University, and a Scientific Manager with Alternative Energies and Atomic Energy Commission (CEA) - National Institute for Solar Energy (INES). His research interests are in the fields of power system analysis, operations, electromagnetic transients, distributed generation, smartgrid and renewable energy. $\mathrm{He}$ is an IEEE senior member.

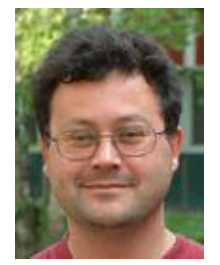

Yvon Besanger received his Ph.D degree in Electrical Engineering from Grenoble Institute of Technology (Grenoble INP), 1996. He is currently Professor at the Engineering School for Energy, Water and Environmental Sciences (ENSE3) and at the Grenoble Electrical Engineering Laboratory (G2Elab) of Grenoble INP, Grenoble Alpes University. His research interests are distribution networks operation and reliability, and power system security.

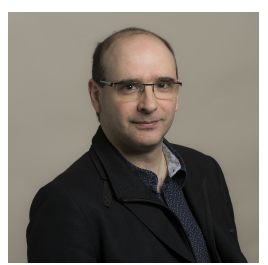

Raphael Caire received the Diplôme d'Etudes Approfondies and Doctorat de l'INPG degrees from the Institut National Polytechnique de Grenoble in 2000 and 2004, respectively. He is currently an Associate Professor with the Grenoble Institute of Technology, Ecole d'ingénieurs en énergie eau et environnement, Grenoble Electrical Engineering Laboratory. His research is centered on the impacts, production control of dispersed generation on distribution system and critical infrastructures. He is an IEEE senior member. 